\title{
An Electrode for Functional Electrical Stimulartion with Solid Polymer Electrolyte
}

\author{
Shigeru YAMAUCHI*, Masasi YAOITA, Haruki IMAZEKI and Yoshihito IKARIYAMA ${ }^{\dagger}$
}

\author{
Received january 26, 1989 ; Accepted March 12, 1990
}

\section{INTRODUCTION}

Patients with injured spinal chord suffer from paralysis in limbs. Their peripheral nerves are still alive and their muscles can work by electrical stimulation. Recent advances in computer technology have initiated development of a therapy called functional electrical stimulation (FES) in which computer controlled electric pulse is applied to peripheral nerves or muscles of paraplegic and/or quadriplegic patients ${ }^{1,2)}$.

Although nerves can be stimulated through surface electrodes, implantable electrodes are necessary for precise control of muscle motion. Normally, platinum-iridium alloy or stainless steel has been employed as an electrode material. Electric current is carried by electrons in the electrodes, whereas cations and anions carry it in the human body. Electrochemical redox reactions of biochemical substances take place at the interface, yielding toxic products.2)

Several attempts have been made to minimize the above mentioned biochemical redox reactions. They are: balanced square wave biphasic pulse, which applies anodic and cathodic pulses with the same amount of charge 1 ). capacitive electrode, whose surface is covered by dielectric materials such as tantalum pentoxide ${ }^{3)}$. double layer charging current by decreasing current density4). platinized platinum electrode by keeping current and charge within a limit of surface oxidation and $\mathrm{H}$-atom plating 5 ).

Since the electrode reactions are irreversible, biphasic pulses cannot eliminate redox reactions completely. Current by utilization of capacitive electrode is also limited by the capacitance of the electrodes. Moreover, Faradaic current cannot be

National Rehabilitation Center for the Disabled, Namiki 4-I, Tolorozawa, Sultama 359

tDopartmont of Bioenginecring, Tokgo Institute of

Tochnology, Ookapame 2-12-1, Moguro-tu, Tokyo 152 neglected even with low current density. At the platinized platinum electrodes biochemical substances such as ascorbate and glucose are easily oxidized at lower potential. Thus none of the above attempts have been able to develop a satisfactory electrode.

Therefore, we consider that the electrode which can supply electric pulses without redox reaction of biochemical substances is indispensable for the development of successful FES systems. We have recently proposed a new electrode system to meet the above contradictory requirement by separating biochemical and electrode systems with solid electrolyte film ${ }^{6}$ ). In this concept only the electrode material is subject to redox reaction and ionic current flows through the solid electrolyte. This paper presents some preliminary results to demonstrate that the above strategy is valid.

\section{EXPERIMENTAL}

We have selected sodium tungsten bronze $\left(\mathrm{Na}_{\mathrm{x}} \mathrm{WO}_{3}, \mathrm{x}=0.2,0.4,0.6,0.8\right.$ and 1.0) as the electrode material, since electrochemical incorporation of sodium ions has already been utilized for electrochromic devices. Moreover, tungsten bronze has been used as a stable electrode for FES 1 ).

Tungsten bronze was prepared by firing a mixture of $\mathrm{Na}_{2} \mathrm{WO}_{4}, \mathrm{WO}_{3}$, and $\mathrm{W}$ for $170 \mathrm{~h}$ at $800^{\circ} \mathrm{C}$ in vacuum. The values of $x$ were then determined from the lattice constant. The electrodes of $10 \mathrm{~mm}$ in diameter were prepared by cold pressing and sintering. The surface of the electrode was covered with a Nafion film, which was substituted with sodium ions in normal saline solution for several days.

The electrode reaction was studied in normal saline solution or in a human serum by cyclic voltammetry at a sweep rate of $1 \mathrm{~V} / \mathrm{s}$ for \pm 0.3 or $1.0 \mathrm{~V}$ around the rest potential. The potentials were recorded against a $\mathrm{Ag} / \mathrm{AgCl}$ reference electrode.

\section{RESULTS}

Cyclic voltammograms in normal saline solution 


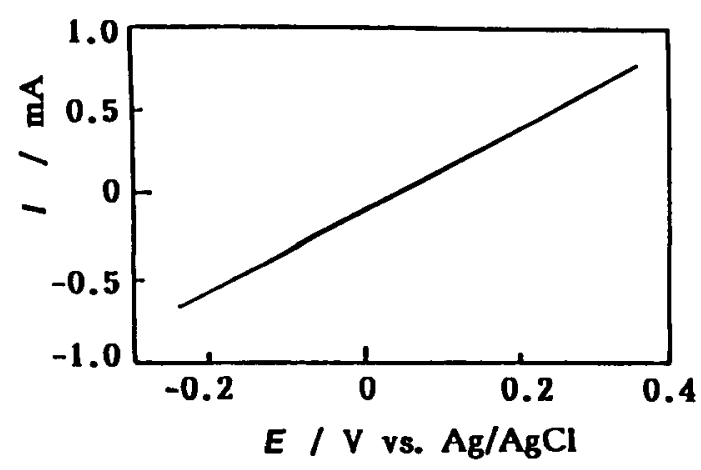

Fig. 1 Cyclic voltammogram of Nafion coated $\mathrm{Na}_{0}{ }_{6} \mathrm{WO}_{3}$ electrode.

gave a current of almost linear dependence on potential, indicating a reversible electrode reaction as illustrated in Fig. 1. The reversible voltammogram in this figure is almost Ohmic and stable for more than 250 cycles. Current at $300 \mathrm{mV}$ of overpotential increased with $\mathrm{x}$, from $0.3 \mathrm{~mA}$ for $\mathrm{x}=0.4$ to $4.5 \mathrm{~mA}$ for $x=1.0$. This dependence implies that tungsten bronze with cubic crystalline structure is favorable for our purpose. It also suggests that the electrode reaction proceeds through incorporation of $\mathrm{Na}^{+}$ions in the Nafion film into $\mathrm{Na}_{x} \mathrm{WO}_{3}$ :

$$
\begin{aligned}
\mathrm{Na}^{+}(\text {in Nafion })+ & \mathrm{e}^{-}\left(\text {in } \mathrm{Na}_{x} \mathrm{WO}_{3}\right) \\
& =\mathrm{Na}\left(\text { in } \mathrm{Na}_{x} \mathrm{WO}_{3}\right)
\end{aligned}
$$

and side reactions are negligible.

It should be also noted that the charge transfer reaction is also reversible and change in non-stoichiometry is small for currents less than 0.5 $\mathrm{mA}$. On the other hand, small hysteresis was observed in the cyclic voltammogram when large currents up to $4.5 \mathrm{~mA}$ was passed through the electrode of $x=1.0$. We consider this hysteresis is a result of change in sodium non-stoichiometry at the surface of the tungsten bronze crystallite and it implies slow diffusion of sodium in the electrode.

The above results clearly indicate that the present strategy to separate the electrode and biological systems by a solid electrolyte provides a novel electrode for electrical stimulation. The electrode material is not necessarily limited to tungsten bronze or related non-stoichiometric compounds. Any redox system such as metal-metal oxide or mixed valence systems could be employed as an electrode material, as long as the charge transfer process is nearly reversible at the required current density. Although mobile ions in the solid electrolyte work as charge carriers, we consider it will be favorable if they are consumed and/or produced by electrode reactions.

In order to demonstrate performance of the

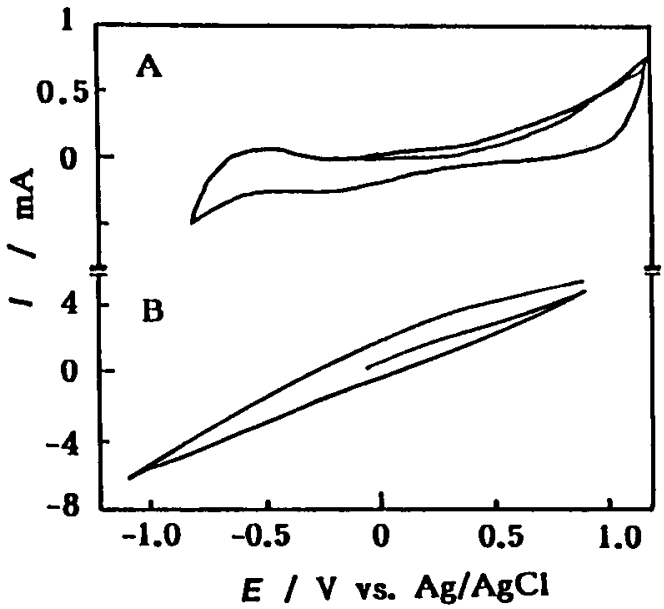

Fig. 2 Cyclic voltammograms in human serum.

A: smooth platinum

B: Nafion coated $\mathrm{Na}_{1.0} \mathrm{WO}_{3}$

present electrode in biological circumstances, we have compared cyclic voltammograms of the present electrode with $\mathrm{x}=1.0$ and platinum electrode in human serum. Curve A in Fig. 2 is a voltammogram of a smooth platinum disk of $10 \mathrm{~mm}$ in diameter and indicates complex redox reactions are involved. On the other hand, the present electrode gave an almost linear voltammogram with some hysteresis, as shown in curve B. We consider the hysteresis is mainly a result of changes in sodium non-stoichiometry at the surface of tungsten bronze, although detailed analysis is required including sodium diffusion.

Summarizing this preliminary report we conclude that the present strategy is promising to develop FES electrodes without any redox reaction of biochemical substances.

\section{References}

1) F. T. Hambrecht and J. B. Reswick Eds., Functional Electrical Stimulation, Marcel Dekker, New York, (1977).

2) J. G. Webster, A. M. Cook, W. J. Tompkins, and G. C. Vanderheiden Eds., Electronic Devices for Rehabilitation, Chapman and Hall, London, (1985).

3) D. L. Guyton and F. T. Hambrecht, Med. Bio. Eng., 12, 613(1974).

4) R. H. Pudentz, Neurosurgery, 1, 190(1977).

5) S. B. Brummer and M. J. Turner, IEEE Tras Biomed. Eng., BME-24, 59(1977).

6) S. Yamauchi, M. Yaoita, Y. Ikariyama, Extended Abstracts, 7th International Conference on Solid State Ionics, p. 208, (1989). 